\title{
Serum Leptin and Risk of Cognitive Decline in Elderly Italians
}

\author{
Thomas J. Littlejohns ${ }^{\mathrm{a}}$, Katarina Kos ${ }^{\mathrm{a}}$, William E. Henley ${ }^{\mathrm{a}}$, Antonio Cherubini ${ }^{\mathrm{b}}$, Luigi Ferrucci ${ }^{\mathrm{c}}$, \\ Iain A. Lang ${ }^{\mathrm{a}}$, Kenneth M. Langa ${ }^{\mathrm{d}, \mathrm{e}}$, David Melzer ${ }^{\mathrm{a}}$ and David J. Llewellyn ${ }^{\mathrm{a}, *}$ \\ ${ }^{a}$ The University of Exeter Medical School, Exeter, UK \\ ${ }^{\mathrm{b}}$ Geriatrics, IRCCS-INRCA, Ancona, Italy (A.C.) \\ ${ }^{\mathrm{c}}$ National Institute on Aging, National Institutes of Health, Baltimore, Maryland, USA \\ ${ }^{\mathrm{d}}$ The Division of General Medicine, Veterans Affairs Ann Arbor Center for Clinical Management Research, \\ Ann Arbor, Michigan, USA \\ ${ }^{\mathrm{e}}$ The Institute for Social Research and the Institute for Healthcare Policy and Innovation, University of Michigan, \\ Ann Arbor, Michigan, USA
}

Accepted 17 October 2014

\begin{abstract}
.
Background: US studies suggest that leptin, a fat-derived hormone, may be protective against the development of dementia. Objective: To investigate the complex relationship between leptin levels and cognitive decline in elderly Italians.

Methods: We studied circulating fasting leptin levels in 809 elderly adults free from dementia who participated in the prospective Italian population-based InCHIANTI study between 1998 and 2009 (mean follow-up of 8.0 years). Global cognitive decline was defined as a reduction of $\geq 5$ points on the Mini-Mental State Examination (MMSE). Trail-Making Tests A and B were also incorporated, with cognitive decline defined as discontinued testing or the worst $10 \%$ of change from baseline. We also investigated whether any association could be explained by midlife weight and whether cognitive decline was associated with changing leptin levels.

Results: The multivariate adjusted relative risk ([RR]; 95\% confidence interval [CI]) of cognitive decline on the MMSE was 0.84 (95\% CI 0.73-0.97) in relation to baseline sex-standardized log-leptin levels. High leptin levels showed a non-significant trend toward a reduced risk of decline on the Trail-Making Tests A $(R R=0.85,95 \% \mathrm{CI} 0.71-1.02)$ and $\mathrm{B}(\mathrm{RR}=0.90,0.79-1.02)$. Adjusting for midlife weight or change in weight did not alter the pattern of results, and cognitive decline was not associated with changing leptin levels.
\end{abstract}

Conclusions: High leptin levels were independently associated with a reduced risk of cognitive decline in elderly Italians.

Keywords: Adipokines, cognitive decline, cohort analysis, epidemiology, leptin

\section{INTRODUCTION}

Leptin is a fat-derived hormone which regulates energy intake and expenditure via hypothalamic signaling [1]. Evidence from in vivo and in vitro studies suggest that leptin is also involved in memory formation and learning [2-4] and reduces the accumulation

${ }^{*}$ Correspondence to: David J. Llewellyn, University of Exeter Medical School, College House, Heavitree Road, Exeter, EX1 2LU, UK. Tel.: +44 1392 726018; E-mail: david.llewellyn@exeter.ac.uk. of amyloid- $\beta[5,6]$ and phosphorylated tau deposits [5], two pathological hallmarks of Alzheimer's disease. Furthermore, leptin levels are significantly lower in individuals with mild cognitive impairment and Alzheimer's disease compared to healthy controls [7]. Several recent prospective studies of elderly US adults have found an association between high leptin levels and a decreased risk of cognitive decline and dementia [8-11]. Stratified analyses suggest that this association may be strongest in non-obese elders [9-11], which is hypothesized to reflect an acquired resistance to 
leptin's neuroprotective effects in obesity [12]. However, no association between midlife leptin and late-life dementia risk was observed in European women [13]. Further studies are therefore necessary to confirm whether midlife and late-life leptin levels are associated with the risk of cognitive decline or dementia in different populations. The possibility of reverse causation remains a key concern that has not been fully addressed by previous studies. In short, early pathological changes associated with dementia may lead to weight loss and a reduction in circulating leptin levels, which in turn may predict an increased risk of subsequent cognitive decline [14].

We therefore investigated the association between serum leptin levels and long-term cognitive decline in a population-based cohort study of elderly Italians. In a series of secondary analyses we also examined whether any association was accounted for by midlife body mass index (BMI), or whether serum leptin levels were changing in participants experiencing cognitive decline.

\section{METHODS}

\section{Population}

The Invecchiare in Chianti (InCHIANTI) study is a population-based prospective cohort study located in Greve in Chianti and Bagno a Ripoli, in Tuscany, Italy and is described in detail elsewhere [15]. 1,270 individuals aged 65 years or older were randomly selected from city registries and 1,155 agreed to participate. We excluded 82 participants with dementia at baseline as diagnosed by geriatricians and a psychologist according to criteria set out in the Diagnostic and Statistical Manual of Mental Disorders (Fourth Edition). Of these, 809 participants had valid baseline fasting serum leptin and cognitive measurements as well as at least one follow-up cognitive assessment conducted in 3 year intervals between 1998 and 2009 (mean [SD] follow-up, 8.0 [2.3] years, median 9.0 years). Compared with the analyzed group, those with missing baseline data or lost to follow-up $(n=266)$ were older (mean [SD] age, 77.8 [8] versus 73.7 [6.6] years old, $p<0.001$ ) and had lower MMSE scores (mean [SD] score, 24 [3.9] versus 25.6 [2.9], $p<0.001$ ). There was no significant difference in serum leptin levels (mean [SD] ng/ml, 13.9 [24] versus 13.6 [16.7], $p=0.86)$, and participants were no more likely to be female $(53.4 \%$ versus $57.4 \%, p=0.26$ ) nor did they differ in years of education (mean [SD], 5.2 [3.7] versus 5.5 [3.2] years, $p=0.14)$.

\section{Standard protocol approvals, registrations, and patient consents}

Ethical approval for the study was provided by the Instituto Nazionale Riposo e Cura Anziani institutional review board. Informed consent to participate was obtained from participants or, if they could not provide consent, a close relative was asked to provide consent on their behalf.

\section{Cognitive tests}

The Mini-Mental State Examination (MMSE) and Trail-Making Tests A and B (Trails A and B) were administered at baseline and repeated at years 3,6, and 9 of follow-up. The MMSE is a widely used screening test for dementia and cognitive decline in the general population that measures various aspects of cognitive functioning including memory, concentration, visuospatial skills, orientation, and language abilities [16]. Overall scores range from 0-30, with lower scores indicating worse cognitive functioning. The Trails A and B provide information about executive functioning, visual search, visual attention, and motor speed [17]. Trails A requires individuals to draw a line between 25 numbers in a sequential order $(1,2,3$, etc.) while Trails B alternates between numbers and letters (1, A, 2, B, etc.). The former places greater emphasis on attention, and the latter places more demand on executive function. Longer times, with a range from $0-300$ seconds, indicate worse cognitive functioning. 675 participants had at least one follow-up attempt at Trails A and 551 participants had at least one follow-up attempt at Trails B.

\section{Leptin assay}

Blood samples were obtained from fasting samples in 1998-2000, 2001-2003, and 2004-2006 and were frozen at $-80^{\circ} \mathrm{C}$ until analysis. Serum leptin levels were determined using a multiplex assay kit (Human Endocrine LINCOplex kit; Linco Research, Inc, St. Charles, MO, USA) and the minimum detectable concentration was $1 \mathrm{ng} / \mathrm{mL}$ with an intra-assay coefficient of variation of $5.1 \%$. Further descriptive data regarding leptin is provided in Supplementary Table 1.

\section{Covariates}

We adjusted for covariates that could potentially confound the relationship between leptin and cognitive decline $[8,9]$. These included age in years, sex, baseline cognitive score, number of years education, depressive symptoms $(\geq 16$ on the Center for 
Table 1

Baseline characteristics of 809 participants stratified by sex-standardized log-leptin quartiles (ng/mL)

\begin{tabular}{|c|c|c|c|c|c|c|}
\hline & \multirow{2}{*}{$\begin{array}{c}\text { All } \\
(n=809)\end{array}$} & \multicolumn{4}{|c|}{ Leptin quartiles } & \multirow[t]{2}{*}{$p$-value } \\
\hline & & $\begin{array}{c}1 \\
(n=203)\end{array}$ & $\begin{array}{c}2 \\
(n=202)\end{array}$ & $\begin{array}{c}3 \\
(n=202)\end{array}$ & $\begin{array}{c}4 \\
(n=202)\end{array}$ & \\
\hline Leptin ng/mL, mean (SD) & $13.6(16.7)$ & $3.2(2.1)$ & $7.5(3.5)$ & $12.8(6.0)$ & $31.0(24.9)$ & $<0.001$ \\
\hline Females, No. (\%) & $464(57.4)$ & $116(57.1)$ & $116(57.4)$ & $116(57.4)$ & $116(57.4)$ & 1 \\
\hline Age in years, mean (SD) & $73.7(6.6)$ & $75.4(7.2)$ & $73.6(6.7)$ & $72.4(6.0)$ & $73.5(6.2)$ & 0.01 \\
\hline Years in school, mean (SD) & $5.5(3.2)$ & $5.4(3.3)$ & $5.6(3.3)$ & $5.6(3.2)$ & $5.4(3.2)$ & 0.8 \\
\hline $\begin{array}{l}\text { CES-D depression score } \geq 16 \text {, } \\
\text { No./total }(\%)\end{array}$ & $125 / 807(15.5)$ & $33 / 202(16.3)$ & $33 / 201(16.4)$ & $31(15.4)$ & $28(13.9)$ & 0.88 \\
\hline $\begin{array}{l}\text { Current cigarette smoker, } \\
\text { No. }(\%)\end{array}$ & $107(13.2)$ & $33(16.3)$ & 24 (11.9) & $26(12.8)$ & $24(11.9)$ & 0.52 \\
\hline Stroke, No./total (\%) & 40/801 (5.0) & $13 / 200(6.5)$ & $4 / 200(2.0)$ & $10 / 201(5.0)$ & $13 / 200(6.5)$ & 0.13 \\
\hline $\begin{array}{l}\text { Cardiovascular disease, } \\
\text { No./total }(\%)\end{array}$ & $115 / 801(14.4)$ & $29 / 200(14.5)$ & $28 / 200(14.0)$ & $28 / 201(13.9)$ & $30 / 200(15.0)$ & 0.99 \\
\hline Hypertension, No./total (\%) & $626 / 801(78.2)$ & $158 / 200(79.0)$ & $151 / 200(75.5)$ & $155 / 201(71.1)$ & $162 / 200(81.0)$ & 0.58 \\
\hline Diabetes, No./total (\%) & $110 / 801(13.7)$ & $21 / 200(10.5)$ & $30 / 200(15.0)$ & 20/201 (10.0) & $39 / 200(19.5)$ & 0.02 \\
\hline \multicolumn{7}{|l|}{ Cognitive scores, mean (SD) } \\
\hline MMSE & $25.6(2.9)$ & $25.4(3.2)$ & $25.8(2.9)$ & $25.7(2.9)$ & $25.5(2.7)$ & 0.39 \\
\hline Trails A & $94.6(60.6)$ & $104.4(69.6)$ & $88.9(57.6)$ & $88.9(56.4)$ & $96.7(57.8)$ & 0.06 \\
\hline Trails B & $175.5(75.8)$ & $171.4(71.2)$ & $171.9(74.3)$ & $180.1(78.7)$ & $177.7(78.3)$ & 0.72 \\
\hline APOE-e4, No./total (\%) & $91 / 591(15.4)$ & $22 / 146(15.1)$ & $26 / 150(17.3)$ & $18 / 147(12.2)$ & $25(16.9)$ & 0.61 \\
\hline Late-life BMI, No./total (\%) & & & & & & $<0.001$ \\
\hline$<25$ & $228 / 790(28.9)$ & $116 / 195(59.5)$ & $70 / 198(33.4)$ & $30 / 200(15.0)$ & $12 / 197(6.1)$ & \\
\hline $25-<30$ & $364 / 790(46.1)$ & $67 / 195(34.4)$ & $99 / 198(50.0)$ & $118 / 200(59.0)$ & $80 / 197(40.6)$ & \\
\hline$\geq 30$ & $198 / 790(25.1)$ & $12 / 195(6.2)$ & 29/198 (14.7) & $52 / 200(26.0)$ & $105 / 197(53.3)$ & \\
\hline Midlife BMI, No./total (\%) & & & & & & $<0.001$ \\
\hline$<25$ & $256 / 709(36.1)$ & $84 / 178(47.2)$ & $69 / 177(38.0)$ & $57 / 182(31.3)$ & $46 / 172(26.4)$ & \\
\hline $25-<30$ & $345 / 709(48.7)$ & $80 / 178(44.9)$ & $85 / 177(48.0)$ & $94 / 182(51.7)$ & $86 / 172(50.0)$ & \\
\hline$\geq 30$ & $108 / 709(15.2)$ & $14 / 178(7.9)$ & $23 / 177(13.0)$ & $31 / 182(17.0)$ & $40 / 172(23.3)$ & \\
\hline Waist-to-hip ratio, mean (SD) & $0.92(0.07)$ & $0.90(0.07)$ & $0.92(0.07)$ & $0.92(0.07)$ & $0.93(0.08)$ & $<0.001$ \\
\hline Years of follow-up, mean (SD) & $7.95(2.29)$ & $7.75(2.32)$ & $7.71(2.58)$ & $8.38(1.91)$ & $7.97(2.25)$ & 0.01 \\
\hline
\end{tabular}

SD, standard deviation; $n$, number of participants; CES-D, Center for Epidemiologic Studies Depression Scale (score of 16 or greater indicates evidence of depressive symptoms); MMSE, Mini-Mental Examination (range, 0-30, higher score represents better cognitive function); Trails A, Trail-Making Test A; Trails B, Trail-Making Test B $p$-value - chi-square for categorical variables and ANOVA for continuous variables.

Epidemiologic Studies Depression Scale), smoking status (current versus non/former smoker), stroke (medical history, physical exam), cardiovascular disease (medical history of myocardial infarction, angina pectoris, peripheral arterial disease, or atrial fibrillation or right carotid stenosis $\geq 40 \%$ measured using carotid ultrasound color Doppler examination), hypertension (medical history, or systolic blood pressure $\geq 140$ $\mathrm{mmHg}$ or diastolic blood pressure $\geq 90 \mathrm{mmHg}$, blood pressure measured with standard mercury sphygmomanometer on three occasions with average of last two measures used in analysis [18]), diabetes (medical history, former or current anti-diabetic treatment, blood glucose levels $\geq 126 \mathrm{mg} / \mathrm{dL}$, glucose measured using an enzymatic colorimetric assay [19]), APOE- $\varepsilon 4$ (present or not present), BMI (groups of $<25,25-<30$ and $\geq 30$, calculated as weight in kilograms divided by height in meters squared) and waist-to-hip ratio (calculated as waist divided by hip in $\mathrm{cm}$ ) and length of follow-up in years.

\section{Midlife BMI}

At study baseline, participants had their height measured in $\mathrm{cm}$ and self-reported their estimated midlife weight (age 50 years) in $\mathrm{kg}$. This information was used to derive midlife BMI $\left(\mathrm{kg} / \mathrm{m}^{2}\right)$. Change in BMI from midlife to baseline was obtained by subtracting midlife BMI from baseline BMI. This was then divided by number of years between age 50 and age at baseline to produce change in BMI from midlife to baseline per year, hereafter referred to as change in BMI. Both midlife BMI and change in BMI were entered into analyses as continuous variables.

\section{Statistical analyses}

Leptin concentration was positively skewed in both sexes with females having significantly higher levels compared to males (mean [SD] ng/ml, 18.4 [19.5] versus 7.2 [8.7], $p<0.001)$. The distribution of leptin 
was therefore normalized using a natural logarithm transformation and then standardized within each sex (mean [SD], 0 [1]). Normally distributed sex standardized leptin was split by quartiles to test for associations with baseline characteristics using analysis of variance for continuous variables and chi-squared test for categorical variables. Multivariate logistic regression models were used to investigate the relationship between baseline sex-standardized log-leptin levels and substantial cognitive decline. Odds ratios were converted to relative risk ratios using the method developed by Zhang and Yu [20]. For the MMSE, substantial cognitive decline was defined as a decrease of 5 points or more from baseline assessment to any stage of follow-up ( $n=221$ decliners) [21]. For Trails A ( $n=144$ decliners) and Trails B ( $n=304$ decliners), substantial cognitive decline was defined as being in the worst $10 \%$ of change from baseline to last followup, or having testing discontinued at last follow-up due to multiple mistakes [22]. There was no evidence of overfitting or collinearity.

The functional form of the relationship between the $\log$ odds of cognitive decline on the MMSE and a smoothing spline plot of sex-standardized logleptin levels was explored using generalized additive models with smoothing splines [23]. This approach provides a method of identifying departures from linearity in exposure-response relationships. Linearity was assessed by visual inspection of the estimated spline functions and by consideration of the "estimated degrees of freedom" for the smoothed terms where values close to 1 were taken as evidence of linearity.

In additional sensitivity analyses we excluded individuals aged over 75 years and with an MMSE score $<24$ at baseline to examine whether there was an association in younger individuals with normal cognitive functioning. To investigate possible effect modification on the basis of body composition, we added interaction terms for leptin X obesity as well as leptin X waist-tohip ratio into the regression models and then performed stratified analysis by BMI groups of normal weight $(<25)$, overweight $(\geq 25-<30)$, and obese $(\geq 30)$ and waist-to-hip ratio (lowest $75 \%$ and highest $25 \%$ ).

In order to investigate the possibility of reverse causation we performed a series of secondary analyses. We repeated the main logistic regression analyses adjusting for BMI at 50 years of age and change in BMI per year from 50 years of age to baseline. We also used a mixed-effects model to investigate differences in sex-standardized log-leptin trajectories from baseline to follow-up measurements at 3 and 6 years between participants who declined and did not decline substantially on the MMSE. Correlations between successive measurements on the same individual were accounted for using parametric models for covariance structure in longitudinal data [24]. Group differences in leptin trajectory were assessed through inclusion of interaction terms between waves of follow-up and cognitive decline group. In an additional analysis, a lagged mixed-effects model was used to investigate whether leptin levels at waves 3 and 6 were associated with MMSE score at baseline and wave 3, respectively. The model was fully adjusted for potential confounders as well as leptin levels at the previous wave. A mixed-effects model was also used to investigate BMI trajectories in participants who declined and did not decline substantially on the MMSE. $P$-values were two-sided throughout and statistical significance was preset at 0.05 . All analyses were performed using Stata/SE version 12.1 (StataCorp, College Station, Texas) with the exception of the spline plot and the leptin and BMI trajectories mixed-effects models which were fitted in $\mathrm{R}$ version 2.15 .1 (http://www.rproject.org).

\section{RESULTS}

Baseline characteristics for participants are provided in Table 1. Significant differences between participants across leptin quartiles were observed for age, where those in the lowest leptin quartile were around two years older than those in quartiles 2-4. Inconsistent differences between quartiles were observed for diabetes and years of follow-up. Measures of waist-tohip ratio and BMI at midlife and late-life increased across leptin quartiles. No differences across leptin quartiles were observed for sex, education in years, depressive symptoms, current smoking status, stroke, cardiovascular disease, hypertension, cognitive scores, or APOE- $\varepsilon 4$ haplotype. Sex-standardized log-leptin levels were highly correlated across waves (baseline to year $3, r=0.64$, baseline to year $6, r=0.60$ and year 3 to year $6, r=0.71$ ).

In basic models adjusted for age, sex, and baseline cognition high leptin levels were significantly associated with a reduced risk of global cognitive decline on the MMSE (Table 2). High leptin was also associated with a reduced risk of cognitive decline on the Trails A and Trails B although this trend did not reach statistical significance. After additional adjustment for major risk factors for cognitive decline, the associations remained highly similar to the minimally adjusted models. The multivariate adjusted relative risk $(95 \%$ 
Table 2

Relative risk of substantial cognitive decline by sex-standardized log-leptin levels ${ }^{\mathrm{a}}$

\begin{tabular}{|c|c|c|c|}
\hline Measure of substantial cognitive decline ${ }^{b}$ & $n$ & $\mathrm{RR}(95 \% \mathrm{CI})$ & $p$-value \\
\hline \multicolumn{4}{|l|}{ MMSE } \\
\hline $\begin{array}{l}\text { Model A: Age, sex and baseline } \\
\text { cognitive score adjusted }\end{array}$ & 809 & $0.85(0.74-0.97)$ & 0.02 \\
\hline $\begin{array}{l}\text { Model B: Model A plus major risk } \\
\text { factors for cognitive decline }\end{array}$ & 799 & $0.84(0.73-0.97)$ & 0.01 \\
\hline $\begin{array}{l}\text { Model C: Model B plus APOE- } 84 \text {, } \\
\text { BMI and waist-to-hip ratio }\end{array}$ & 569 & $0.73(0.59-0.90)$ & 0.003 \\
\hline \multicolumn{4}{|l|}{ Trails A } \\
\hline $\begin{array}{l}\text { Model A: Age, sex and baseline } \\
\text { cognitive score adjusted }\end{array}$ & 675 & $0.85(0.71-1.01)$ & 0.06 \\
\hline $\begin{array}{l}\text { Model B: Model A plus major risk } \\
\text { factors for cognitive decline }\end{array}$ & 673 & $0.85(0.71-1.02)$ & 0.08 \\
\hline $\begin{array}{l}\text { Model C: Model B plus APOE- } \varepsilon 4 \text {, } \\
\text { BMI and waist-to-hip ratio }\end{array}$ & 484 & $0.75(0.57-0.98)$ & 0.03 \\
\hline \multicolumn{4}{|l|}{ Trails $B$} \\
\hline $\begin{array}{l}\text { Model A: Age, sex and baseline } \\
\text { cognitive score adjusted }\end{array}$ & 551 & $0.89(0.78-1.01)$ & 0.07 \\
\hline $\begin{array}{l}\text { Model B: Model A plus major risk } \\
\text { factors for cognitive decline }\end{array}$ & 551 & $0.90(0.79-1.02)$ & 0.11 \\
\hline $\begin{array}{l}\text { Model C: Model B plus APOE- } \varepsilon 4 \text {, } \\
\text { BMI and waist-to-hip ratio }\end{array}$ & 399 & $0.84(0.69-1.00)$ & 0.05 \\
\hline
\end{tabular}

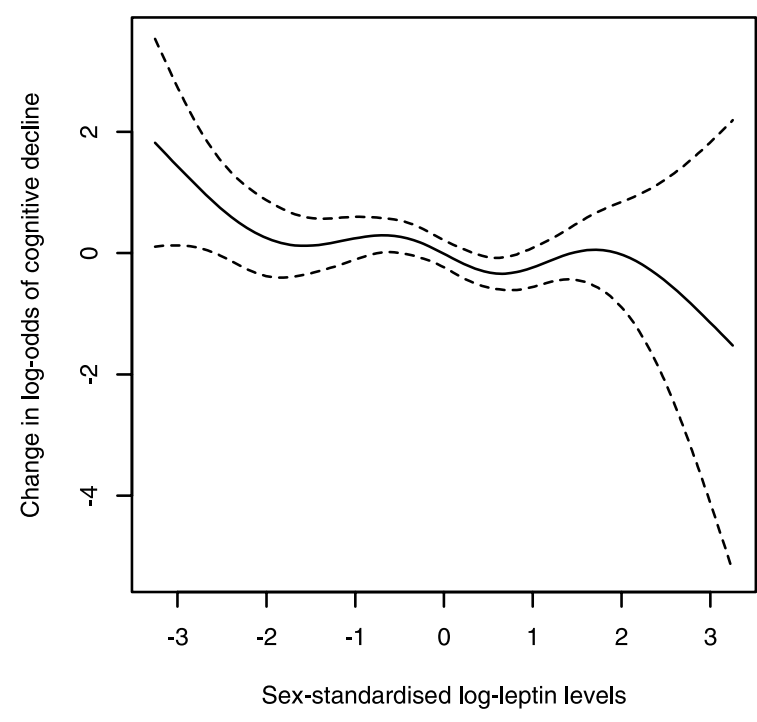

Fig. 1. Smoothing spline plot of sex-standardized log-leptin levels versus change in log odds of cognitive decline on the Mini-Mental State Examination ${ }^{\mathrm{a}, \mathrm{b}}$. ${ }^{\mathrm{a}}$ Leptin was normalized using a natural logarithm transformation and then standardized within each sex (mean [SD], 0 [1]). ${ }^{\mathrm{b}}$ Adjusted for age, sex, baseline cognitive score, years in school, depressive symptoms, smoking, stroke, cardiovascular disease, hypertension, diabetes, and length of follow-up. Substantial cognitive decline defined as a drop of 5 points or more on the MMSE (range, 0-30, higher score represents better function). confidence interval $[\mathrm{CI}]$ ) of cognitive decline on the MMSE was 0.84 (95\% CI 0.73-0.97) in relation to sex-standardized log-leptin levels. High leptin remained associated with a lower risk of cognitive decline on the Trails A and Trails B in fully adjusted models, although again these trends were not statistically significant. Furthermore, the pattern of associations was not altered when adjusting for additional covariates; APOE- 8 4, BMI, and waist-tohip ratio, although the trend for Trails A and Trails B became statistically significant (Table 2). Fig. 1 further illustrates how higher leptin levels are linked with lower odds of global cognitive decline.

When excluding those aged over 75 years and with a baseline MMSE score $<24$, the association between leptin and decline on the MMSE was highly similar to the main findings $(n=458, \mathrm{RR}=0.79,95 \% \mathrm{CI}$ $0.62-1.00, p=0.05$ ).

When including interaction terms in the main analyses, no significant leptin $\mathrm{X}$ obesity interactions were observed (MMSE $p=0.19$; Trails A $p=0.78$; TrailMaking Test $\mathrm{B} p=0.47)$. There was a significant leptin $\mathrm{X}$ waist-to-hip ratio interaction for the MMSE $(p=0.03)$, but not the Trails A $(p=0.21)$ or Trails B $(p=0.81)$. High leptin was associated with a lower risk of cognitive decline on the MMSE and Trails A 
Table 3

Relative risk of substantial cognitive decline by sex-standardized log-leptin levels adjusted for midlife BMI ${ }^{\mathrm{a}}$

\begin{tabular}{|c|c|c|c|c|c|}
\hline \multirow[t]{2}{*}{$\begin{array}{l}\text { Measure of substantial } \\
\text { cognitive decline }{ }^{b}\end{array}$} & \multirow[b]{2}{*}{$n$} & \multicolumn{2}{|c|}{$\begin{array}{l}\text { Adjusted for BMI } \\
\text { at } 50 \text { years old }\end{array}$} & \multicolumn{2}{|c|}{$\begin{array}{c}\text { Adjusted for change } \\
\text { in BMI from } 50 \text { years } \\
\text { old to baseline }\end{array}$} \\
\hline & & $\mathrm{RR}(95 \% \mathrm{CI})$ & $p$-value & $\operatorname{RR}(95 \% \mathrm{CI})$ & $p$-value \\
\hline $\mathrm{MMSE}^{\mathrm{c}}$ & 709 & $0.78(0.66-0.92)$ & 0.002 & $0.82(0.69-0.97)$ & 0.02 \\
\hline Trails $\mathrm{A}^{\mathrm{c}}$ & 611 & $0.85(0.69-1.03)$ & 0.1 & $0.84(0.68-1.03)$ & 0.09 \\
\hline Trails B ${ }^{c}$ & 507 & $0.88(0.76-1.01)$ & 0.08 & $0.89(0.76-1.02)$ & 0.09 \\
\hline
\end{tabular}

$\mathrm{RR}$, relative risk ratio; $\mathrm{CI}$, confidence interval; BMI, body mass index. ${ }^{a}$ Leptin was normalized using a natural logarithm transformation and then standardized within each sex (mean [SD], 0 [1]). ${ }^{\text {b Substantial cognitive decline }}$ defined as a drop of 5 points or more on the MMSE (range, 0-30, higher score represents better function) and the worst $10 \%$ of cognitive change or test discontinued for the Trails A and B. ${ }^{\mathrm{c}}$ Adjusted for age, sex, baseline cognitive score, years in school, depressive symptoms, smoking, stroke, cardiovascular disease, hypertension, diabetes and length of follow-up.

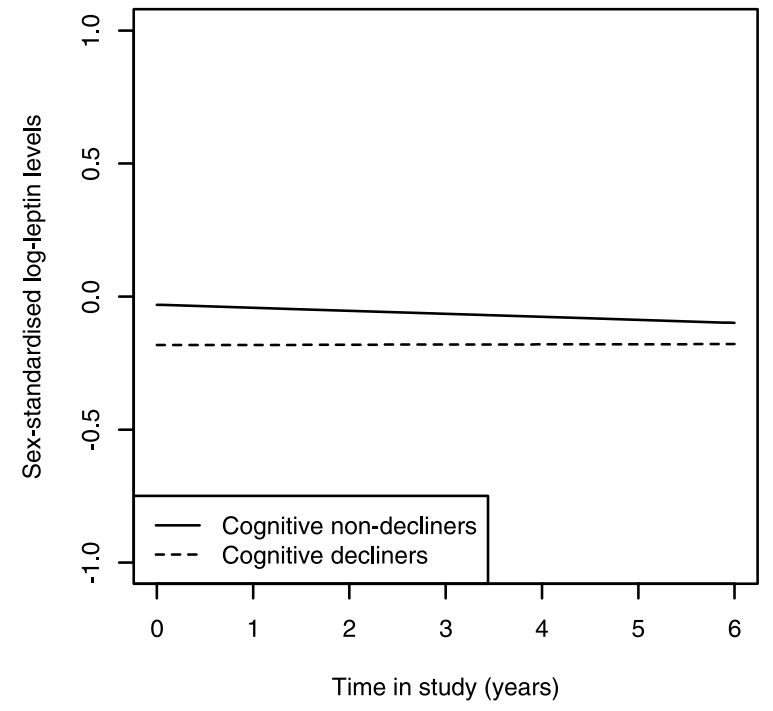

Fig. 2. Mixed effects model of change in sex-standardized log-leptin levels over 6 years by cognitive decliners and cognitive non-decliners on the Mini-Mental State Examination ${ }^{\mathrm{a}, \mathrm{b}, \mathrm{c}}$. ${ }^{\mathrm{a}}$ Leptin was normalized using a natural logarithm transformation and then standardized within each sex (mean [SD], 0 [1]). ${ }^{\mathrm{b}}$ Adjusted for age, sex, baseline cognitive score, years in school, depressive symptoms, smoking, stroke, cardiovascular disease, hypertension, diabetes, and length of follow-up. Substantial cognitive decline defined as a drop of 5 points or more on the MMSE (range, 0-30, higher score represents better function). ${ }^{\mathrm{c}}$ Cognitive non-decliners, $n=584$; Cognitive decliners, $n=215$.

and $\mathrm{B}$ when analyses were restricted to overweight participants (BMI $\geq 25-<30$ ), and the MMSE when restricted to those in the lowest three quartiles of waistto-hip ratio (Supplementary Table 2). There were no significant associations between leptin levels and cognitive decline in normal weight or obese participants or those in the highest waist-to-hip ratio quartile.

Additional adjustment for midlife BMI or change in BMI from midlife to baseline made little difference to the pattern of associations (Table 3 ). For example, after adjustment for BMI at 50 years of age, the relative risk of cognitive decline on the MMSE was 0.78 (95\% CI 0.66-0.92). A mixed-effects model found that the trajectories of serum leptin between baseline and follow-up did not differ significantly between participants who declined substantially on the MMSE and those who did not ( $p=0.34$, Fig. 2). Additionally, a lagged mixed-effects model found that leptin levels at wave 3 and 6 were not associated with MMSE score at baseline or wave $3(\beta=-0.01,95 \% \mathrm{CI}-0.03-0.00$, $p=0.10$ ). A further mixed-effects analysis found that BMI declined significantly more between baseline and follow-up in participants who declined on the MMSE compared to those who did not $(p=0.002)$.

\section{DISCUSSION}

In an Italian prospective population-based cohort study of elderly dementia-free adults, higher leptin levels at baseline were associated with a lower risk of global cognitive decline. This association remained after adjustment for potential confounders including demographic variables and vascular risk factors. Adjustment for midlife BMI or change in BMI did not account for the association, and cognitive decline did not predict subsequent changes in circulating leptin, reducing the likelihood of reverse causation. Conversely, cognitive decline predicted significant decrease in BMI throughout follow-up as expected [14]. Excluding over 75 year olds with low cognitive function at baseline did not change the pattern of results. Stratification on the basis of body composition revealed that the association was strongest in overweight participants.

Our findings are consistent with recent prospective studies conducted in elderly adults in the US that have found an association between high leptin levels and a 
reduced risk of cognitive decline and dementia [8-11]. Our study is the first to demonstrate that the association is found in a non-US population despite a different pattern of potential confounders. Previous studies were also unable to take into account midlife body composition and did not incorporate multiple measures of circulating leptin to address the possibility of reverse causation.

One study observed significant interactions between obesity and waist-to-hip ratio and all-cause dementia; with high leptin associated with a lower risk of dementia in the non-obese and those in the lowest three quartiles of waist-to-hip ratio [9]. Similarly, another study found higher leptin associated with a lower risk of cognitive decline in adults with a small waist circumference but no association in those with a large waist circumference [10]. However, a different study found that high leptin levels were associated with a reduced dementia risk in normal weight elderly women, but there was no association in those overweight or obese [11]. Our results suggest that the association may be strongest in overweight elderly adults, though there was no interaction between leptin and obesity for any of the cognitive measures. There was evidence for possible effect modification by waist-to-hip ratio for global cognitive decline, but not for attention or executive function. Despite methodological differences, there is little evidence for an association between leptin and dementia-related outcomes in elderly adults who are obese or have a large waist circumference. This is hypothesized to reflect an acquired resistance to the central effects of leptin, though this remains poorly understood [25-27]. Given the inconsistent findings for normal weight and overweight elderly adults, further research is needed to address this.

While late-life leptin levels seem to be associated with dementia risk, there was no evidence for an association between midlife leptin levels and late-life dementia risk in Swedish women followed for 31 to 35 years [13]. This raises the possibility that reverse causation leads to an association between late-life leptin and dementia-related outcomes due to declining leptin levels and weight loss linked with neuropathological changes [14], or that midlife leptin levels are a poor indicator of overall leptin levels during follow-up or late-life leptin levels due to regression dilution bias [28]. Our results make the former less likely by demonstrating that the associations observed in late-life are not accounted for by midlife BMI or change in BMI. Furthermore leptin levels were stable in older adults who experienced substantial cognitive decline, again making reverse causation less likely.
Cellular and animal research provides compelling evidence that leptin has multiple neuroprotective effects. Leptin was found to facilitate hippocampal synaptic plasticity via NMDA receptors in rats, which are necessary for the induction of long-term potentiation [3]. Leptin increases motility and density of dendritic filopodia in hippocampal neurons which is associated with a rapid increase in the formation of hippocampal synaptic connections [29]. Rodents with leptin receptor deficiency in the hippocampus demonstrate poor spatial memory and impaired long term potentiation [30]. Direct injection of leptin into the hippocampus improves memory performance in a transgenic animal model of Alzheimer's disease [2]. Leptin reduces the accumulation of the Alzheimer's disease hallmarks amyloid- $\beta[5,6]$ and phosphorylated tau [5]. Furthermore leptin may reduce both hallmarks through AMPK and sirtuin pathways as well as the inhibition of glycogen synthase kinase-3 $\beta$ [31-34].

Our study had a number of strengths; to our knowledge this is the first study outside of the US to investigate the association between late-life leptin levels and cognitive decline. The sample is a reasonably large population-based sample, and attrition during follow-up was low. It is the first study to adjust for midlife weight and to examine changes in late-life leptin levels in relation to cognitive decline. These analyses allowed us to address the possibility of reverse causation in a more robust way than has previously been possible. We restricted our analyses to those with high levels of cognitive function at baseline which did not alter the pattern of associations. Our study had a number of limitations. The InCHIANTI cohort is limited to participants of elderly white individuals, so our findings may not generalize to other populations. Statistical power for Trails A and B analyses was comparatively limited due to the smaller number of participants who completed these tests. Midlife BMI was derived from midlife weight by self-report and height measured at baseline and is therefore less accurate than if BMI was measured directly at midlife. As with all observational studies, unmeasured confounding remains a possibility, and our findings do not establish a causal relationship.

We found that high serum leptin levels were prospectively associated with a reduced risk of global cognitive decline in elderly Italians. This association remained significant in models fully adjusted for a variety of potential confounders. This is the first study to confirm that recent findings from the US generalize to another population [8-11]. Adjustment for midlife weight did not change the pattern of associations, and 
leptin levels were stable in those experiencing cognitive decline making reverse causation less likely. Further studies are therefore warranted to investigate whether leptin has relevance to the prevention or treatment of Alzheimer's disease and other dementia subtypes.

\section{ACKNOWLEDGMENTS}

The InCHIANTI study baseline (1998-2000) was supported as a "targeted project" (ICS110.1/RF97.71) by the Italian Ministry of Health, and in part by the U.S. National Institute on Aging (Contracts: 263 MD 9164 and 263 MD 821336). The follow-up 1 (2001-2003) was funded by the U.S. National Institute on Aging (Contracts: N.1-AG-1-1 and N.1-AG-12111); the follow-up 2 and 3 studies (2004-2010) were financed by the U.S. National Institute on Aging (Contract: N01-AG-5-0002). Supported in part by the Intramural research program of the National Institute on Aging, National Institutes of Health, Baltimore, Maryland. Additional support was also provided by NIRG-11-200737 from the Alzheimer's Association, the Mary Kinross Charitable Trust, the James Tudor Foundation, the Halpin Trust, the Sir Halley Stewart Trust, the Age Related Diseases and Health Trust, and the Norman Family Charitable Trust (to D.J.L.). This research was supported by the National Institute for Health Research (NIHR) Collaboration for Leadership in Applied Health Research and Care South West Peninsula at the Royal Devon and Exeter NHS Foundation Trust. The views expressed are those of the authors and not necessarily those of the NHS, the NIHR or the Department of Health. None of the funding sources had any role in the design of the study; in the analysis and interpretation of the data; or in the preparation of the manuscript.

Authors' disclosures available online (http://www.jalz.com/disclosures/view.php?id=2609).

The authors have no conflicts of interest to declare.

\section{SUPPLEMENTARY MATERIAL}

The supplementary material is available in the electronic version of this article: http://dx.doi.org/10.3233/ JAD-141836.

\section{REFERENCES}

[1] Friedman JM, Halaas JL (1998) Leptin and the regulation of body weight in mammals. Nature 395, 763-770.
[2] Farr S, Banks W, Morley JE (2006) Effects of leptin on memory processing. Peptides 27, 1420-1425.

[3] Shanley LJ, Irving AJ, Harvey J (2001) Leptin enhances NMDA receptor function and modulates hippocampal synaptic plasticity. J Neurosci 21, RC186.

[4] Weigle DS, Duell PB, Connor WE, Steiner RA, Soules MR, Kuijper JL (1997) Effect of fasting, refeeding, and dietary fat restriction on plasma leptin levels. J Clin Edocrinology Metab 82, 561-565.

[5] Marwarha G, Dasari B, Prasanthi JRP, Schommer J, Ghribi O (2010) Leptin reduces the accumulation of Abeta and phosphorylated tau induced by 27-hydroxycholesterol in rabbit organotypic slices. J Alzheimers Dis 19, 1007-1019.

[6] Fewlass DC, Noboa K, Pi-Sunyer FX, Johnston JM, Yan SD, Tezapsidis N (2004) Obesity-related leptin regulates Alzheimer's A $\beta$. FASEB J 18, 1870-1878.

[7] Johnston JM, Hu WT, Fardo DW, Greco SJ, Perry G, Montine TJ, Trojanowski JQ, Shaw LM, Ashford JW, Tezapsidis $\mathrm{N}$ (2014) Low plasma leptin in cognitively impaired ADNI subjects: Gender differences and diagnostic and therapeutic potential. Curr Alzheimer Res 11, 165-174.

[8] Holden KF, Lindquist K, Tylavsky FA, Rosano C, Harris TB, Yaffe K (2009) Serum leptin level and cognition in the elderly: Findings from the Health ABC Study. Neurobiol Aging 30, 1483-1489.

[9] Lieb W, Beiser AS, Vasan RS, Tan ZS, Au R, Harris TB, Roubenoff R, Auerbach S, DeCarli C, Wolf PA, Seshadri S (2009) Association of plasma leptin levels with incident Alzheimer disease and MRI measures of brain aging. JAMA 302, 2565-2572.

[10] Zeki Al Hazzouri A, Haan MN, Whitmer RA, Yaffe K, Neuhaus J (2012) Central obesity, leptin and cognitive decline: The sacramento area latino study on aging. Dement Geriatr Cogn Disord 33, 400-409.

[11] Zeki Al Hazzouri A, Stone KL, Haan MN, Yaffe K (2013) Leptin, mild cognitive impairment, and dementia among elderly women. J Gerontol A Biol Sci Med Sci 68, 175-180.

[12] Considine RV, Sinha MK, Heiman ML, Kriauciunas A, Stephens TW, Nyce MR, Ohannesian JP, Marco CC, McKee LJ, Bauer TL (1996) Serum immunoreactive-leptin concentrations in normal-weight and obese humans. $N$ Engl J Med 334, 292-295.

[13] Gustafson DR, Bäckman K, Lissner L, Carlsson L, Waern M, Ostling S, Guo X, Bengtsson C, Skoog I (2012) Leptin and dementia over 32 years-The Prospective Population Study of Women. Alzheimers Dement 8, 272-277.

[14] Stewart R, Masaki K, Xue Q-L, Peila R, Petrovitch H, White LR, Launer LJ (2005) A 32-year prospective study of change in body weight and incident dementia: The Honolulu-Asia Aging Study. Arch Neurol 62, 55-60.

[15] Ferrucci L, Bandinelli S, Benvenuti E, Di Iorio A, Macchi C, Harris TB, Guralnik JM (2000) Subsystems contributing to the decline in ability to walk: Bridging the gap between epidemiology and geriatric practice in the InCHIANTI study. J Am Geriatr Soc 48, 1618-1625.

[16] Folstein MF, Folstein SE, McHugh PR (1975) Mini-mental state. A practical method for grading the cognitive state of patients for the clinician. J Psychiatr Res 12, 189-198.

[17] Reitan RM (1958) Validity of the Trail Making Test as an indicator of organic brain damage. Percept Mot Skills 8, 271-276.

[18] Maggio M, Lauretani F, Ceda GP, Bandinelli S, Basaria S, Paolisso G, Giumelli C, Luci M, Najjar SS, Metter EJ, Valenti G, Guralnik J, Ferrucci L (2010) Estradiol and metabolic syndrome in older italian men: The InCHIANTI Study. J Androl 31, 155-162. 
[19] Maggio M, Lauretani F, Ceda GP, Bandinelli S, Basaria S, Paolisso G, Ble A, Egan JM, Metter EJ, Abbatecola AM, Zuliani G, Ruggiero C, Valenti G, Guralnik JM, Ferrucci L (2007) Association of hormonal dysregulation with metabolic syndrome in older women: Data from the InCHIANTI study. Am J Physiol Endocrinol Metab 292, E353-E358.

[20] Zhang J, Yu KF (1998) What's the relative risk? A method of correcting the odds ratio in cohort studies of common outcomes. JAMA 280, 1690-1691.

[21] Gustafson DR, Skoog I, Rosengren L, Zetterberg H, Blennow K (2007) Cerebrospinal fluid beta-amyloid 1-42 concentration may predict cognitive decline in older women. $J$ Neurol Neurosurg Psychiatry 78, 461-464.

[22] Llewellyn DJ, Lang IA, Langa KM, Muniz-Terrera G, Phillips CL, Cherubini A, Ferrucci L, Melzer D (2010) Vitamin D and risk of cognitive decline in elderly persons. Arch Intern Med 170, 1135-1141.

[23] Wood S (2006) Generalized additive models: An introduction with $R$, Chapman and Hall/CRC.

[24] Diggle P, Liang K-Y, Zeger SL (1994) Analysis oflongitudinal data, Clarendon Press.

[25] Banks WA, DiPalma CR, Farrell CL (1999) Impaired transport of leptin across the blood-brain barrier in obesity. Peptides 20, 1341-1345.

[26] Banks WA, Coon AB, Robinson SM, Moinuddin A, Shultz JM, Nakaoke R, Morley JE (2004) Triglycerides induce leptin resistance at the blood-brain barrier. Diabetes 53, 1253-1260

[27] Zhang Y, Scarpace PJ (2006) The role of leptin in leptin resistance and obesity. Physiol Behav 88, 249-256.
[28] Clarke R, Shipley M, Lewington S, Youngman L, Collins R, Marmot M, Peto R (1999) Underestimation of risk associations due to regression dilution in long-term follow-up of prospective studies. Am J Epidemiol 150, 341-353.

[29] O'Malley D, MacDonald N, Mizielinska S, Connolly CN, Irving AJ, Harvey J (2007) Leptin promotes rapid dynamic changes in hippocampal dendritic morphology. Mol Cell Neurosci 35, 559-572.

[30] Li X-L, Aou S, Oomura Y, Hori N, Fukunaga K, Hori T (2002) Impairment of long-term potentiation and spatial memory in leptin receptor-deficient rodents. Neuroscience 113, 607-615.

[31] Greco SJ, Sarkar S, Johnston JM, Zhu X, Su B, Casadesus G, Ashford JW, Smith MA, Tezapsidis N (2008) Leptin reduces Alzheimer's disease-related tau phosphorylation in neuronal cells. Biochem Biophys Res Commun 376, 536-541.

[32] Greco SJ, Sarkar S, Casadesus G, Zhu X, Smith MA, Ashford JW, Johnston JM, Tezapsidis N (2009) Leptin inhibits glycogen synthase kinase- $3 \beta$ to prevent tau phosphorylation in neuronal cells. Neurosci Lett 455, 191-194.

[33] Greco SJ, Sarkar S, Johnston JM, Tezapsidis N (2009) Leptin regulates tau phosphorylation and amyloid through AMPK in neuronal cells. Biochem Biophys Res Commun 380, 98-104.

[34] Greco SJ, Hamzelou A, Johnston JM, Smith MA, Ashford JW, Tezapsidis N (2011) Leptin boosts cellular metabolism by activating AMPK and the sirtuins to reduce tau phosphorylation and $\beta$-amyloid in neurons. Biochem Biophys Res Commun 414, 170-174. 